\title{
Cervical Cancer- Care Delayed-Care Denied and Death Invited
}

\section{Navya ${ }^{1 *}$ and Suresh $\mathrm{K}^{2}$}

${ }^{1}$ B. Sc (AT), MPH, School of Environmental Science, Public Health \& Sanitation Management, Karnataka State Rural Development and Panchayat Raj University, India ${ }^{2}$ MD. DIH, FIAP, FIPHA, FISCD, Freelance Public Health Consultant \& Visiting Professor KSRDPRU, GADAG, Karnataka, India

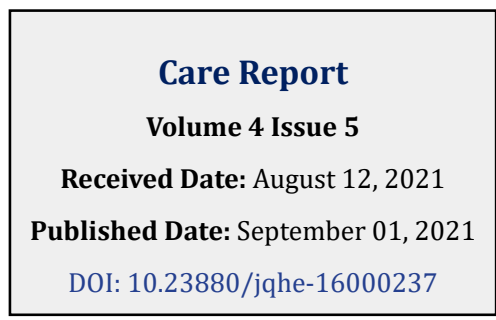

*Corresponding author: Navya MS, KSRDPRU, Gadag, Karnataka, Pin 582102, India, Tel: 917483359683; Email: navyareddy199820@gmail.com

\section{Abstract}

With about 78 billion global population, in 2020, 19 million new cancers and nearly 1 million deaths due to all types estimated to occur. Cervical cancer does not figure in top 5 most common cancers in both sexes globally, but with $6.5 \%$ incidence it forms the fourth top among women worldwide. The cancer registry data across the country in India with simple average of the most recent local rates applied to 2020 population estimates a figure of 1324413 cases and 851678 in 2020 and cumulative prevalence around 2.7 million cases in last 5 years of all categories of cancers in India. Cervical cancers in India account for $10 \%$ of all cancers in both sexes and about $18 \%$ of cancers among women in India as per the international agency for Cancer Research. Nearly one-third of the global cervical cancer deaths occur in India. It is a preventable condition as it has a long precancerous stage amenable for screening and treating. Cervical cancer Cervix uteri is no 3 with about 123907 cases (9.45) next only to Breast and Oral cancers with a 2.01 cumulative risk. Cervical cancer death risk as 60000 women die annually. While the incidence and mortality rates of cervical cancer in the world are 13.1/ and 6.9/ per lakh population, in India, it is estimated to be 14.7/and 9.2/ per lakh population. Resulting in about 96,922 new cervical cancer cases and 60,078 deaths ranking second among women cancers. The incidence varies within Indian population basically due to ggeographical and socio-economic disparities in screening practices and care seeking practices across the districts of India.

Epidemiologically the symptoms are mild and mimic a venereal disease of low infectiousness and therefore delay in seeking care and reporting at a time when the prognosis is poor. The 5 -year survival rate is around $76.0 \%$ for those aged $\leq 50$ years with much poorer survival among cases with involvement lymph nodes. We present one such case of a 45 year's old rural women reporting on 4th May 2021 with symptoms vaginal bleeding, back pain, urine leakage \& pelvic pain since 2 months to a gynecologic oncology service at a cancer hospital \& research center, Bangalore. She had the history of taking treatment for another 2 months earlier from a traditional healer with no progress. She had consulted a family doctor first who referred her to the Cancer hospital. After an evaluation for abnormal vagina bleeding, cervical biopsies that demonstrated invasive adenocarcinoma of the cervix (Stage IV B CA Cervix) the diagnosis was confirmed. Since it was in an advanced stage, a palliative management including radiation therapy for control of bleeding \& pain, and systemic chemotherapy for disseminated disease was opted. After 22 days of treatment she got discharged along with medications prescribed Inj. Amikacin 100mg IV OD, Inj. Pantoprazole 40mg IV OD, DNS which was given by the local doctor. But there was no progress and finally she died on 17 th June 2021 after 23 days of discharge. This case emphasizes the need for early care seeking behavior, regular screening for all women over 35-45 years and HPV vaccination for adolescent girls in India.

Keywords: Cervical Cancer; Screening; Late Stage; Metastasis; Radiotherapy and Chemotherapy; Rural Women; Lack of Awareness; Belief in Traditional Treatments 


\section{Introduction}

With about 78 billion global population, 19 million new cancers and nearly 1 million deaths due to all types of cancers estimated to occur in 2020. Cervical cancer does not figure in top 5 most common cancers in both sexes globally, but with $6.5 \%$ incidence it forms the fourth top among women worldwide. The cancer registry data across the country in India with simple average of the most recent local rates applied to 2020 population estimates a figure of 1324413 cases and 851678 deaths in 2020 and cumulative prevalence around 2.7 million cases in last 5 years of all categories of cancers in India [1]. Cervical cancers in India account for $10 \%$ of all cancers in both sexes and about $18 \%$ of cancers among women in India as per the international agency for Cancer Research (Globocan 2020). Nearly onethird of the global cervical cancer deaths occur in India. It is a preventable condition as it has a long pre-cancerous stage amenable for screening and treating. Cervical cancer Cervix uteri is no 3 with about 123907 cases (9.45\%) next only to Breast and Oral cancers with a 2.01 cumulative risk. While the incidence and mortality rates of cervical cancer in the world are 13.1/ and 6.9/ per lakh population, in India, it is estimated to be 14.7 /and 9.2 / per lakh population. This will be about 96,922 new cervical cancer cases and 60,078 deaths ranking second among women cancers in 2020 [2]. India is experiencing a rapid health transition with a rising burden of Non-Communicable Diseases (NCD) surpassing the burden of Communicable diseases. Cervical cancer is the leading cause of cancer deaths in women in India. The human papillomavirus (HPV) is responsible for virtually all cervical cancers has given way for developing a vaccine for preventing this disease [3-7]. Though cervical cancer is on the declining trend according to the population-based registries; its screening has not yet penetrated rural India and it continues to be a major public health problem for women in India. Multifactorial causation, potentials for prevention, and the sheer threat it poses, make cervical cancer an important disease for in-depth studies and countrywide interventions [4]. Disparities of access to health care and poverty play a major role in high mortality rate. To control and prevent major NCDs, the National Programme for Prevention and Control of Cancer, Diabetes, Cardiovascular Diseases and Stroke (NPCDCS) was launched in 2010 with focus on strengthening infrastructure, (HRD) human resource development, health promotion, early diagnosis, management, and referral [2]. Nationwide education, promotion screening services are still short due to lack of awareness about the importance and urgency among rural and slum population and inaccessibility of such services in subdistrict level. The routine human papillomavirus (HPV) testing and vaccination can reduce in cervical cancer deaths. Studies on HPV DNA testing and the low-technology method of "screen and treat" are promising. In addition, decreasing the cost and increasing the availability of HPV vaccines in developing nations brings hope and promise to the next generation of women.

Cervical cancer can be prevented by doing PAP smear screening and getting HPV vaccine done. Symptoms include bleeding in between periods and also after sexual intercourse. Foul smelling white discharge, low backache and lower abdominal pain may also occur. In some cases there may not be any symptoms. Almost all cervical cancers are caused by human papillomavirus. HPV is a common virus that spread through skin-to-skin contact and by sexual intercourse. Treatments include surgery, radiation, and also chemotherapy (Figure 1).

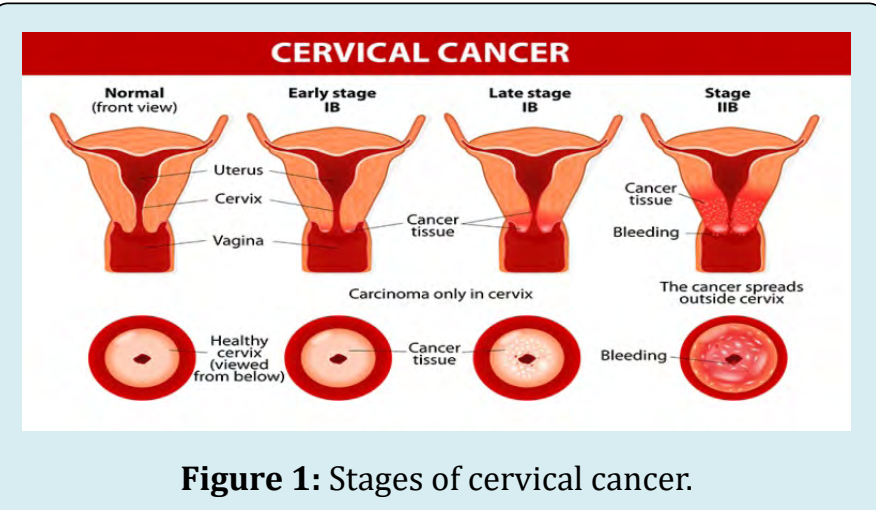

\section{Case Presentation}

45-year-old rural women, with 3 children having never had a smear test/screening tests earlier in life was referred by a family doctor on $4^{\text {th }}$ May 2021 with symptoms of vaginal bleeding, pelvic discomfort, back pain, leakage of urine for 2 months to the gynecologic oncology service at Oncoville cancer hospital \& research center, Bangalore. She had the history of taking treatment for 2 months earlier from a traditional healer with no improvement.

\section{Physical Examinations}

- Pulse $72 / \mathrm{min}$

- $\quad$ BP $135 / 85 \mathrm{mmhg}$

- Temperature 98.20F

- $\mathrm{SPO}_{2}$ 97\% (mandated during Covid 19 Pandemic)

- Weight 44kgs

\section{Investigations}

A battery of tests like blood sugar, cholesterol, LFT, THYROID hormones\& serological RA factors were normal, RFT was abnormal \& $\mathrm{Hb} \%$ was low(8dl/mg).

Colposcopy examination indicated involvement rectum 
and Lymph nodes.

\section{Diagnosis}

Based on the investigations results, a positive colposcopy results she was diagnosed as having 'Stage IVB cervical cancer' having affected lymph nodes a very late stage.

\section{Treatment}

Radiation therapy with chemotherapy was done for 3 weeks to slow down the growth of cancer \& help relieve symptoms. Patient was discharged from hospital along with prescription of - Inj. Amikacin 100mg IV OD, Inj. pantoprazole 40mg IV OD, Inj. Diclofenac IV OD, \& DNS which were given by the family doctor.

\section{Progress}

Sadly, there was no progress in patient's condition, as she continued to bleed, and lose weight. Patient got swelling in her hands \& legs in her last day.

\section{Outcome}

Finally, she died on $17^{\text {th }}$ June 2021 after 23 days of discharge from hospital.

\section{Discussion}

Regular smear test can discover the sign of precancerous cell and treat the patient according to the result. The Classification for Pap smear Analysis aims at classifying the Pap smear cells whether it is affected or not by using the extracted features of grey level co-occurrence matrix technique. Cervical cancer stages use in India cancer program are [8].

- Stage 1: Cancer has formed and is found in the cervix only.

- Stage 2: The cancer has spread to the upper part of the vagina or the tissue surrounding the uterus, but it hasn't spread to lymph nodes or distant sites.

- Stage 3: The cancer has spread to the lower third of the vagina and/or to the pelvic wall and/or has caused kidney problems, and/or involves lymph nodes.

- Stage 4: The cancer has spread beyond the pelvis or has spread to the lining of the bladder or rectum or has spread to other parts of the body.

- Stage 4A: The cancer has grown into nearby organs such as the bladder or rectum or is spreading out of the pelvic area.

- Stage 4B: The cancer has spread to the liver, lungs, bones, or distant lymph nodes.
Cervical cancer is one of the leading causes of cancer death among females worldwide, more often a silent killer. As observed in this case cervical cancer was diagnosed at very late stage and not treatable as she was symptomatic only for about 4 months. The cancer had spread out of the pelvis to other areas of the body in the first consultation itself. Indian Cancer Society standard treatment protocols were used in this case that included radiation therapy with chemotherapy to slow the growth of the cancer \& help relieve symptoms [8].

This case emphasizes the need for regular screening for all women over 35-45 years and HPV vaccination for adolescent girls and early care seeking behavior in India. Many women avoiding PAP smears screening is not simply a matter of personal choice or being irresponsible. Other reasons include psychological factors like fear, discomfort, social stigma about cervical cancer, distrust of medical services \& faith in traditional healing methods. Rural people approach traditional healers more for the treatment [10].

If diagnosed at an early stage cervical cancers are curable. These cancers are preventable with access to periodic screening tests, high-quality care, and regular follow-up [8]. The strategies to reduce the high burden of cervical cancers include risk factor intervention, screening, vaccination, and early diagnosis [11].

Cancer prevention with the help of a vaccine is a comparatively new concept, awareness and education will have important implication in the implementation of this strategy. A study after giving awareness about HPV vaccine among target population, seen increased overall acceptance of HPV vaccine to $67.8 \%$ [12].

A cross sectional study done in south India on screening and awareness among women indicated that very few women are screened as only few had adequate knowledge of the disease. This study suggested community-based education followed by screening campaign is the need of the time to reduce the prevalence of cervical cancer in population [1114].

\section{Conclusion}

Despite being one of the top Cancers among women in India, and national program since 2010 many women are dying due to lack of awareness, accessibility of services of the disease especially in rural \& tribal areas. To add to the problem belief in traditional healing methods because of accessibility, affordability is influencing delayed seeking appropriate care. The national NCD screening program has not yet widely spread and influenced creating awareness about screening activities \& HPV vaccine. As even the educated girls are neither aware nor vaccinated, starting an 
exclusive mandatory vaccination for school and college girls is the need of the time.

\section{Take Home Message}

1. Popularizing HPV vaccine for girls in the age group of 9-25years is a priority.

2. Increasing accessibility for screening tests of cervical cancer through H\&WCs.

3. Avoiding unsafe sex \& smoking.

\section{References}

1. India facts sheet.

2. Balasubramaniam G (2020) Survival rate of cervical cancer from a study conducted in India. pp: 1-10.

3. National Program for prevention \& Control of Cancer, Diabetes, Cardiovascular Diseases \& stroke (NPCDCS).

4. A Sreedevi (2015) Epidemiology of cervical cancer with special focus on India. Int J Women's Health 7: 405-414.

5. Walboomers JM, Jacobs MV, Manos MM, Bosch FX, Kummer JA, et al. (1999) Human papillomavirus is a necessary cause of invasive cervical cancer worldwide. J Pathol 189(1): 12-19.

6. Bosch FX, Lorincz A, Munoz N, Meijer CJ, Shah KV (2002)
The causal relation between human papillomavirus and cervical cancer. J Clin Pathol 55(4): 244-265.

7. Munoz N (2003) Epidemiologic classification of human papillomavirus types associated with cervical cancer. $\mathrm{N}$ Engl J Med 348(6): 518-527.

8. National cancer institute.

9. Cervical cancer Treatment guidelines.

10. Kakar DN (1983) Traditional healers in North India: a study. Nurs J India 74(3): 61-33.

11. WHO (2017) WHO Cancer Resolution; Cancer prevention and control in the context of an integrated approach. $17^{\text {th }}$ World Heal Assem pp: 1-6.

12. Monica (2020) An epidemiological study of cervical and breast screening in India.

13. Pandey D, Vanya V, Bhagat S, Vs B, Shetty J (2012) Awareness, and attitude towards human papillomavirus vaccine among medical students in a premier medical school in India. PLoS One 7(7): e40619.

14. Alyse R (2020) Prevalence of Cervical Cancer Screening and Awareness among Women in an Urban Community in South India-A Cross Sectional Study. Annals of global health 86(1): 130-116. 